\title{
Interaction of E3 Ubiquitin Ligase MARCH7 with Long Noncoding RNA MALAT1 and Autophagy-Related Protein ATG7 Promotes Autophagy and Invasion in Ovarian Cancer
}

\author{
Jianguo $\mathrm{Hu}^{\mathrm{a}}$ Luo Zhang ${ }^{\mathrm{a}}$ Zhiqiang Meic Yuan Jiang ${ }^{\mathrm{d}}$ Yuan Yie Li Liu \\ Ying Meng ${ }^{\mathrm{a}}$ Lili Zhou $^{\mathrm{b}}$ Jianhua Zeng ${ }^{\mathrm{a}}$ Huan $\mathrm{Wu}^{\mathrm{a}}$ Xingwei Jiang ${ }^{\mathrm{a}}$
}

\begin{abstract}
aDepartment of Obstetrics and Gynecology, Second Affiliated Hospital, Chongqing Medical University, Chongqing, 'Department of cardiology, Second Affiliated Hospital, Chongqing Medical University, Chongqing, 'The Research Center for Preclinical Medicine, Southwest Medical University, Luzhou, dDepartment of Rehabilitation Medicine,The First Affiliated Hospital of Chengdu Medical College, Chengdu, eDepartment of Orthopaedics, Traditional Chinese Medicine Hospital, Southwest Medical University, Luzhou, P.R.China
\end{abstract}

\section{Key Words}

March7 • MALAT1 • ATG7 • Autophagy $・ S m a d 2 / 3 \cdot$ Ovarian cancer • Invasion

\begin{abstract}
:
Background/Aims: Ubiquitin E3 ligase MARCH7 plays an important role in T cell proliferation and neuronal development. But its role in ovarian cancer remains unclear. This study aimed to investigate the role of Ubiquitin E3 ligase MARCH7 in ovarian cancer. Methods: Real-time $\mathrm{PCR}$, immunohistochemistry and western blotting analysis were performed to determine the expression of MARCH7, MALAT1 and ATG7 in ovarian cancer cell lines and clinical specimens. The role of MARCH7 in maintaining ovarian cancer malignant phenotype was examined by Wound healing assay, Matrigel invasion assays and Mouse orthotopic xenograft model. Luciferase reporter assay, western blot analysis and ChIP assay were used to determine whether MARCH7 activates TGF- $\beta$-smad2/3 pathway by interacting with TGF $\beta$ R2. Results: MARCH7 interacted with MALAT1 by miR-200a (microRNA-200a). MARCH7 may function as a competing endogenous RNA (ceRNA) to regulate the expression of ATG7 by competing with miR-200a. MARCH7 regulated TGF- $\beta$-smad $2 / 3$ pathway by interacting with TGF $\beta R 2$. Inhibition of TGF- $\beta$-smad2/3 pathway downregulated MARCH7, MALAT1 and ATG7. MiR-200a regulated TGF- $\beta$ induced autophagy, invasion and metastasis of SKOV3 cells by targeting MARCH7. MARCH7 silencing inhibited autophagy invasion and metastasis of SKOV3 cells both in vitro and in vivo. In contrast, MARCH7 overexpression promoted TGF- $\beta$ induced autophagy, invasion and metastasis of A2780 cells in vitro by depending on MALAT1 and ATG7. We also found that TGF- $\beta$-smad2/3 pathway regulated MARCH7 and ATG7 through MALAT1. Conclusions: These findings suggested that TGF $\beta$ R2-Smad2/3-MALAT1/MARCH7/ATG7 feedback loop mediated autophagy, migration and invasion in ovarian cancer.

\begin{tabular}{ll}
\hline Xingwei Jiang & Department of Obstetrics and Gynecology, Second Affiliated Hospital, Chongqing Medical University \\
& Chongqing (China) \\
& E-Mail angeljianguo@163.com
\end{tabular}
\end{abstract}




\section{Cellular Physiology Cell Physiol Biochem 2018;47:654-666 \begin{tabular}{c|c|c|} 
DOI: 10.1159/000490020 & $\begin{array}{l}\text { O } 2018 \text { The Author(s). Published by S. Karger AG, Basel } \\
\text { www.karger.com/cpb }\end{array}$ \\
\cline { 2 - 3 }
\end{tabular}}

Hu et al.: MARCH7 Promotes Autophagy and Invasion in Ovarian Cancer

\section{Introduction}

Transforming growth factor-beta (TGF- $\beta$ ) is the prototypical member that belongs to a large family of cytokines. It regulates various cellular functions such as proliferation, differentiation, migration, apoptosis, adhesion, angiogenesis, immune surveillance, and survival of various types of cells. Similar to the duality of autophagy in cancer, TGF- $\beta$ serves as both tumor suppressor and tumor promoter $[1,2]$. During the early phase of carcinogenesis, TGF- $\beta$ acts as a tumor suppressor inhibiting the primary tumor development and growth by inducing cell cycle arrest and apoptosis.

However, in the late stages of tumor progression, some tumor cells become resistant to growth inhibition by TGF- $\beta$, and TGF-beta here acts as a tumor promoter. TGF- $\beta$ facilitates the progression and metastasis of tumors in advanced cancer [3-6]. Recent studies have showed that TGF- $\beta$ induced autophagy in normal bovine mammary epithelial BME-UV1 cells and some cancer cells [7]. Activation of autophagy by TGF- $\beta$ potentiates TGF- $\beta$-mediated growth inhibition in human hepatocellular carcinoma cells. Inhibition of c-Jun NH2-terminal kinase or knockdown of Smad2/3, Smad4 or DAPK attenuates TGF- $\beta$ induced autophagy. This indicates that both Smad and non-Smad pathways are involved in the process. It has been reported that autophagy promotes migration in cancer cells [8]. However, the role of autophagy activation by TGF- $\beta$ in migration and invasion of cancer cells have not been elucidated.

Our previous study has demonstrated that E3 ubiquitin ligase MARCH7 promotes ovarian cancer growth, and also observed that TGF- $\beta$ increased the mRNA and protein expression of MARCH7 [9]. However, the mechanisms of MARCH7 upregulation by TGF- $\beta$ and its role in autophagy, migration and invasion of cancer cells have not been elucidated yet. Hence, in the present study, we aimed to evaluate the interaction of MARCH7 with MALAT1 and ATG7 in the regulation of TGF- $\beta$ induced autophagy, migration and invasion through TGF- $\beta$-smad $2 / 3$ pathway.

\section{Materials and Methods}

Tissue specimens

The tissue microarray slides containing malignant and benign ovarian tissues $(n=77)$ were obtained from US Biomax Inc cancer tissue bank collection (US Biomax Inc., MD, USA). The Ethics Committee of the Chongqing Medical University approved the study documents and the use of archived cancer tissues.

Cell culture, transfection procedure, and reagents

Human ovarian cancer cells were cultured in Rosewall Park Memorial Institute (RPMI) 1640 medium, containing $10 \%$ fetal bovine serum and antibiotics, and incubated at $37^{\circ} \mathrm{C}$ in $5 \%$ carbon dioxide atmosphere. Double-strand oligonucleotides corresponding to the target sequences were synthesized by Genepharma Co., Ltd. (Shanghai, China). The following sequences were targeted for human MARCH7, MALAT1, and ATG7 small interfering RNA (siRNA), respectively. MARCH71:5'-GCACUUGGGAGUAAUUUGA-3'; MARCH7-2:5'-GCACACGUGUCCGAUUUAU-3'; $\quad$ MALAT1: 5'-CACAGGGAAAGCGAGTGGTTGGTAA-3'; ATG7: 5'-CAGAAGGAGUCACAGCUCUUCCUUA-3'; and NC (negative control) siRNA: 5'-UUCUUCGAAGGUGUCACGUTT-3'. Lentiviral vector expressing shRNA targeting MARCH7 (named LV3- shMarch7-1 and LV3-shMarch7-2) and MARCH7-lentiviral expression vector (named LV5-March7) were provided from Genepharma Co., Ltd. (Shanghai, China). MiR-200a mimics (sense: 5'-UAACACUGUCUGGUAACGAUGU-3') were synthesized at Ruibo Biotech (Ruibo Biotechnology, Guangzhou, China).

In situ hybridization for MALAT1

In Situ Hybridization (ISH) was performed according to the MALAT1 In Situ Hybridization (ISH) Kit instructions (MK3588, Boster Co., Ltd., Wuhan, China). 


\section{Cellular Physiology Cell Physiol Biochem 2018;47:654-666 and Biochemistry Published onIIne: IVlay 25, $2018 \quad$\begin{tabular}{l|l} 
DOI: 10.1159/000490020 2018 The Author(s). Published by S. Karger AG, Basel \\
www.karger.com/cpb
\end{tabular}}

Hu et al.: MARCH7 Promotes Autophagy and Invasion in Ovarian Cancer

\section{Immunohistochemistry}

Immunohistochemistry (IHC) was performed according to the SP kit instructions (SP-9000, ZSGB-BIO, Beijing, China). After dewaxing and hydration, the sections were heated in citrate buffer (pH 6.0, SigmaAldrich, USA) in a microwave oven for 20 minutes for antigen retrieval. Furthermore, the sections were cooled naturally to room temperature. The sections were washed thrice for 3 minutes per cycle. Subsequently, the sections were incubated in 3\% aquae hydrogenii dioxidi for 15 minutes at room temperature, and washed thrice with phosphate buffered saline (PBS) for 3 minutes per cycle. The sections were blocked with 5\% goat serum (Bioss Biotechnology) for 30 minutes at $37^{\circ} \mathrm{C}$. Anti-MARCH7 rabbit polyclonal antibody (1:100, bs-9341R, Bioss, Beijing, China), and anti-ATG7 rabbit monoclonal antibody (1:100, ab52472, abcam) were incubated with the sections overnight at $4^{\circ} \mathrm{C}$. Negative controls included omission of primary antibody and use of irrelevant primary antibodies. The corresponding secondary antibodies that were conjugated to horseradish peroxidase (Bioss Biotechnology) were incubated with the sections for an hour at room temperature. The sections were washed thrice in PBS for 3 minutes per cycle. The sections were incubated in horseradish enzyme-labeled chain avidin solution (Bioss Biotechnology) for 30 minutes at $37^{\circ} \mathrm{C}$, and washed in PBS for 3 minutes $x 3$ cycles. The proteins were visualized by diaminobenzidine. The staining data were obtained from manually recorded reports. Staining intensity was graded on a 0-3 scale as follows: 0 (absence of staining), 1 (weakly stained), 2 (moderately stained), and 3 (strongly stained). The percentage of positive tumor cells was scored as follows: 0 (absence of tumor cells), 1 ( $<33 \%$ tumor cells), 2 (33-66\% tumor cells) and 3 (>66\% tumor cells). Immunohistochemical score (ranging from 0 to 9 ) was calculated by multiplying the intensity score and the percentage score [10].

\section{Quantitative real-time polymerase chain reaction (PCR)}

Total RNA was isolated using an RNA pure High-purity Total RNA Rapid Extraction Kit (BioTeke, RP1201, China), as per the instructions provided in the kit. cDNA was synthesized using the iSCRIPT cDNA synthesis kit (Bio-Rad). The primers used for amplifying MARCH7, ATG7, MALAT1 and GAPDH were synthesized by Guangzhou Funeng Co., Ltd. The real-time PCR kit was purchased from Guangzhou Funeng Co., Ltd. PCR conditions were $95^{\circ} \mathrm{C}$ for 10 seconds, $60^{\circ} \mathrm{C}$ for 20 seconds, $72^{\circ} \mathrm{C}$ for 10 seconds. Each sample was analyzed in triplicate. Relative quantification of mRNA was performed using the comparative threshold cycles (CT) method. This value was used to plot the gene expression employing the formula $2^{-\Delta \Delta C T}$.

\section{Detection of protein expression by Western blotting}

Expression of P-SMAD2, ATG7, MARCH7 and GAPDH proteins was analyzed by Western blotting $[11,12]$. The primary antibodies used included polyclonal rabbit anti-MARCH7 (1:500, bs-9341R, Bioss, Beijing, China); Rabbit monoclonal to p-Smad2 (1:1000; ab188334; Abcam Inc., Cambridge, MA, USA); AntiATG7 rabbit monoclonal antibody (1:1000, ab52472, abcam), and polyclonal rabbit anti-GAPDH (1:1000; AB10016; Sangon Biotech, Shanghai, China). The band density was analyzed using a gel imaging system and compared against an internal control.

\section{Dual-luciferase reporter gene assay}

Luciferase reporter gene assay was performed using the Dual-Luciferase Reporter Assay System (Promega) according to the instructions provided by the manufacturer. For MARCH7, ATG7 and MALAT1 3' UTR luciferase reporter assay, wild type or mutant reporter constructs (termed WT or Mut; purchased from Genepharma Co., Ltd., Shanghai, China) were co-transfected into SKOV3 cells in 24-well plates with 100 nM miR-200a or 100 nM miR-NC and Renilla plasmid by using Endofectin ${ }^{\text {TM }}$-Plus (GeneCopoeia). SMAD2/3 reporter plasmid was purchased from Shanghai Qcbio Science \& Technologies Co., Ltd. (Shanghai, China). Reporter gene assay was performed 48 hours post-transfection using the Dual Luciferase Assay System (Promega, Madison, WI). Firefly luciferase activity was normalized for transfection efficiency using the corresponding Renilla luciferase activity. All experiments were performed at least 3 times.

\section{Chromatin immunoprecipitation}

Chromatin immunoprecipitation (ChIP) was carried out by using the Chromatin Immunoprecipitation (ChIP) Assay Kit (Beyotime Biotechnology) according to the manufacturer's protocol. Briefly, cross-linked chromatin was sonicated into 200-to 1, 000-bp fragments. Then, the chromatin was immunoprecipitated using anti-SMAD2/3 antibody. Quantitative PCR was conducted according to the method described above. 


\section{Cellular Physiology Cell Physiol Biochem 2018;47:654-666 and Biochemistry Publisned onIIne: IVlay 25, $2018 \quad$\begin{tabular}{l|l} 
DOI: 10.1159/000490020 2018 The Author(s). Published by S. Karger AG, Basel \\
www.karger.com/cpb
\end{tabular}}

Hu et al.: MARCH7 Promotes Autophagy and Invasion in Ovarian Cancer

Wound healing assay and Matrigel invasion assays

Migration of SKOV3 and A2780 cells were analyzed using the wound-healing assay in vitro. Cells were seeded into 6-well plates and cultivated until 90\% growth confluence. Wounds were afflicted by scraping the monolayer cells with a sterile pipette tip. At 0 and 48 hours after the wounding, cells were observed under low power in an Olympus light microscope. The distance between the 2 wounds were measured at each time point, and expressed as the average percent of wound closure as compared to that at zero time. Invasion of SKOV3 and A2780 cells were evaluated by Matrigel invasion assays. For Transwell invasion assays, the upper side of an $8 \mu \mathrm{m}$ pore, $6.5-\mathrm{mm}$ polycarbonate transwell filter (Corning, New York, NY) chamber was uniformly coated with Matrigel basement membrane matrix (BD Biosciences, Bedford, MA) for $2 \mathrm{~h}$ at $37^{\circ} \mathrm{C}$ before the cells were added. A total of $5 \times 10^{4}$ cells were seeded into the top chamber of a trans-well filter (in triplicate) and incubated for 48 hours. Invasive cells on the lower side of the filter were fixed in $4 \%$ paraformaldehyde, stained in $0.5 \%$ crystal violet (Beyotime), and counted using a microscope. A total of 5 fields were counted for each transwell filter. Each field was counted and photographed at 200x magnification.

\section{Mouse orthotopic xenograft model of ovarian cancer}

The xenograft model was prepared as described previously [13]. All procedures for animal experiments were approved by the Committee on the Use and Care on Animals (Chongqing Medical University, Chongqing, China), and performed in accordance with the institution guidelines. Ovarian cancer SKOV3 cells were infected with LV3-shMARCH7-1 or LV3-NC, then injected intraperitoneally (i.p.) into 6-week-old BALB/c nude mice $\left(5 \times 10^{6}\right.$ cells per mouse in 200 ul). Five weeks after i.p. injection, animals were sacrificed to confirm the presence of tumors and weigh the established tumors. After sacrifice, ascites was quantified, the number of metastases was counted and carefully dissected, and the removed tumor was then weighed.

\section{Statistical analysis}

All statistical analyses were performed using SPSS software, version 17.0 (Chicago, IL). Each experiment was performed in triplicates. Statistical analysis was performed by Student's t-test or analysis of variance (ANOVA). The correlation between MARCH7, MALAT1 and ATG7 expression was analyzed by using ChiSquare test. Data were presented as mean \pm standard deviation. Statistical significance was defined as a p-value less than 0.05 .

\section{Results}

MARCH7 interaction with MALAT1 depends on miR-200a

Bioinformatics analyses (http://bioinfo.bjmu.edu.cn/lncpro/, http://string905.embl. de/newstring_cgi/show_network_section.pl) showed that MARCH7 may interact with MALAT1. We firstly detected the expression of MALAT1 in ovarian cancer and normal tissues. The expression of MALAT1 was increased in ovarian cancer tissues compared with the normal ovarian tissues (Fig. 1A). We then analyzed the correlation between MARCH7 and MALAT1 by detecting the expression of MARCH7 and MALAT1 in ovarian cancer tissues and cell lines. Our results indicated that MARCH7 expression was correlated well with MALAT1 expression (Fig. 1B; correlation coefficient is 0.89).

As reported, LncRNA could regulate the target protein mainly by directly binding to the target protein [14]. Thus, to further validate the association between MALAT1 and MARCH7, we performed RNA immunoprecipitation (RIP) assay with an antibody against MARCH7 on SKOV3 cellular extracts. However, we did not observe any significant higher enrichment level of MALAT1 with MARCH7 antibody compared with nonspecific IgG control antibody (data not shown). Based on these results, we concluded that MALAT1 may not directly regulate the expression of MARCH7. We hypothesized that MALAT1 might regulate MARCH7 through posttranscriptional modification. After detailed discussion of the regulatory mechanism of LncRNA that was described previously, we proposed that miRNA might participate in the interaction of MARCH7 and MALAT1. We conducted bioinformatics analysis for the prediction of co-regulation of miRNA on both MARCH7 and MALAT1 by applying multiple databases, 


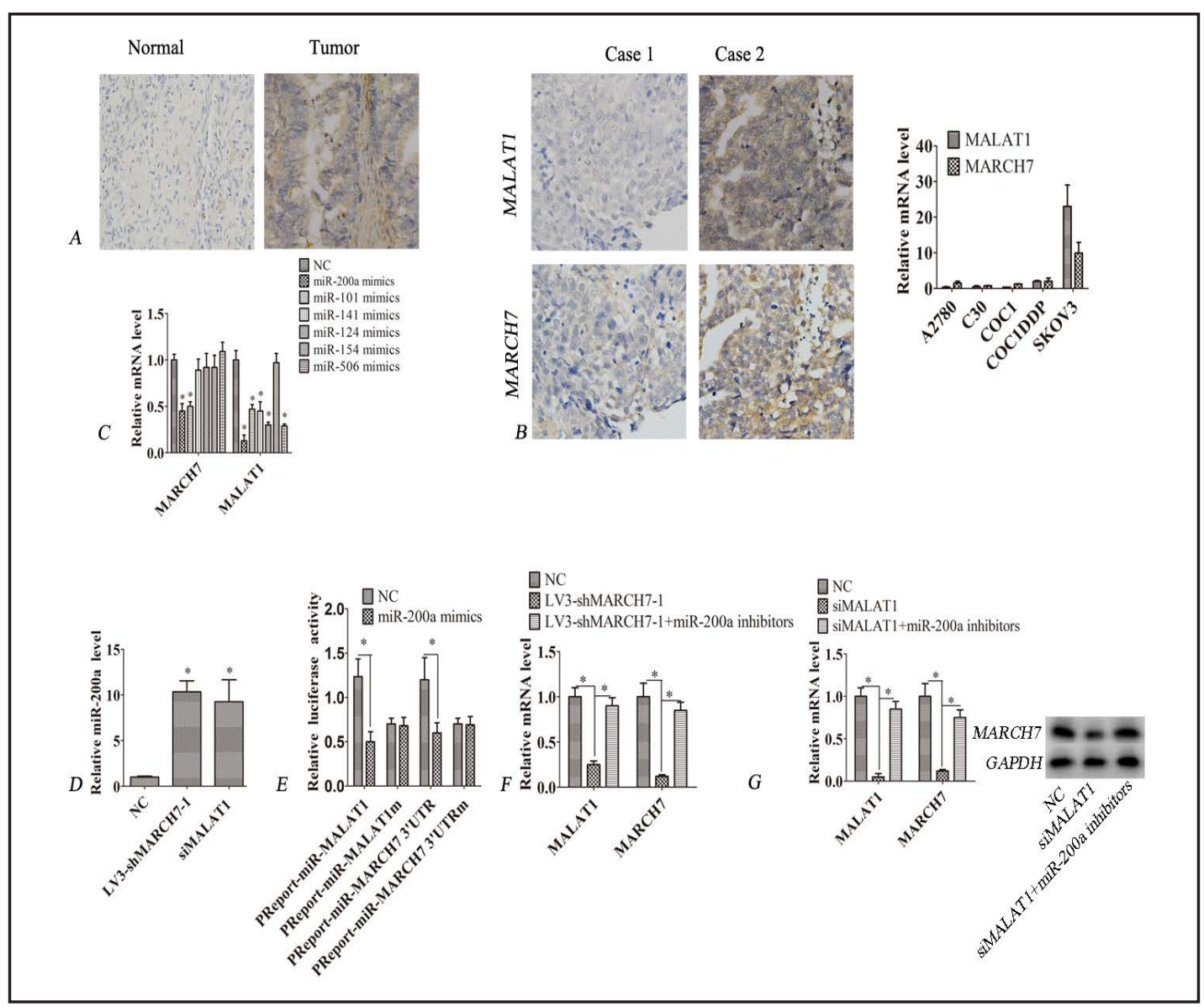

Fig. 1. MARCH7 interacted with MALAT1 depend on miR-200a. (A) In Situ Hybridization (ISH) analysis of MALAT1 expression in ovarian cancer and normal ovarian tissues. (B) Immunohistochemical analysis of MARCH7 and ISH analysis of MALAT1 expression in ovarian cancer. qPCR detected the expression of MALAT1 and MARCH7 in ovarian cancer cell lines. (C) qPCR detected the expression of MALAT1 and MARCH7 when transfected with miR-200a mimic, miR-101 mimic, miR-141 mimic, miR-124 mimic, miR154 mimic and miR-506 mimic in SKOV3 cells. (D) The expression of miR-200a was increased after silencing MARCH7 or MALAT1. (E) SKOV3 cells were co-transfected with miR-200a mimic or control RNA (NC) with luciferase reporter plasmids containing either wild-type (pMIR-MARCH7-3UTR and pMIR-MALAT1) or mutant (pMIR-MARCH7-3UTRm and pMIR-MALAT1m) of MARCH7 and MALAT1 genes. Luciferase expression was measured. The fold changes of the relative luciferase activity in miR-200a mimic indicated that plasmid transfected cells were normalized to NC, indicating plasmid transfected cells. (F) MALAT1 was inhibited when silencing MARCH7. (G) MARCH7 was inhibited when silencing MALAT1. This regulation was abolished when transfected with miR-200a inhibitors. Error bars represent standard error. The symbols * and ${ }^{* *}$ indicate $\mathrm{p}<0.05$ and 0.01 , respectively. Scale bar: $100 \mathrm{um}$.

including BiBi Serv (http://bibiserv.techfak.uni-bielefeld.de/bibi/Tools_RNA_Studio.html), starbase (http://starbase.sysu.edu.cn/), Targetscan (http://www.targetscan.org), PicTar (http://pictar.mdc-berlin.de) and miRbase (http://www.mirbase.org). Six miRNAs (miR200a, miR-141, miR-101, miR-124, miR-154 and miR-506) were selected as candidates. MiR-200a significantly down-regulated the mRNA level of MARCH7 and MALAT1 (Fig. 1C). Silencing of MALAT1 or MARCH7 up-regulated miR-200a (Fig. 1D). Luciferase reporter gene assay was used to confirm the binding of miR-200a with MALAT1 and MARCH7 (Fig. 1E). MALAT1 mRNA showed decreased expression with MARCH7 silencing (Fig. 1F); and MARCH7 mRNA and protein levels were decreased with MALAT1 silencing, whereas this could be reversed when miR-200a was inhibited (Fig.1G). This data indicated that MALAT1 
functions as a competing endogenous RNA in regulating MARCH7 expression by sponging miR-200a in ovarian cancer.

\section{ATG7 acts as a ceRNA of MARCH7}

Bioinformatics analyses (http://starbase.sysu.edu.cn/) showed that ATG7 may function as a competing endogenous RNA to regulate MARCH7 expression. Firstly, we analyzed the correlation between MARCH7 and ATG7 by detecting the expression of MARCH7 and ATG7 in ovarian cancer tissues. The expression of ATG7 was more obvious in tumor tissues as compared with normal ovarian tissues (Fig. 2A). In addition, its expression level was correlated well with MARCH7 expression (Fig. 2B; correlation coefficient is 0.76). MARCH7 silencing down-regulated ATG7 expression. When miR-200a was silenced, this regulation was abolished (Fig. 2C).

Interestingly, miR-200a was a putative miRNA shared by MARCH7 and ATG7. The expression of ATG7 and MARCH7 was decreased when transfected with miR-200a mimic in SKOV3 cells (Fig. 2D). Inhibitor of miR-200a increased the expression of MARCH7 and ATG7 in A2780 cells (Fig. 2E). Luciferase reporter gene assay confirmed the binding of miR-200a with ATG7 (Fig. 2F). This data suggested that ATG7 functions as a competing miR-200a in the regulation of MARCH7 expression.

\section{MALAT1 reciprocally regulated ATG7 dependent miR-200a}

Based on the results above, we investigated whether MALAT1 reciprocally regulated ATG7 dependent miR-200a. Silencing of MALAT1 downregulated the expression of ATG7,

Fig. 2. ATG7 acts as a ceRNA of MARCH7. (A) Immunohistochemical analysis of ATG7 expression in ovarian cancer and normal ovarian tissues. (B) Immunohistochemical analysis of ATG7 and MARCH7 expression in ovarian cancer tissues. (C) ATG7 was inhibited when silencing MARCH7. This regulation was abolished when transfected with miR-200a inhibitors. (D) The expression of MALAT1, MARCH7 and ATG7 was inhibited when transfected with miR-200a mimics. (E) The expression of MALAT1, MARCH7 and ATG7 was increased when transfected with miR-200a inhibitors. (F) SKOV3 cells were cotransfected with miR-200a mimics or control RNA (NC) with luciferase reporter plasmids containing either wild-type pMIR-ATG7-3UTR or mutant pMIR-ATG7-3UTRm of ATG7 gene. Luciferase expression was measured. The fold changes of the relative luciferase activity in miR-200a mimics indicated that the plasmid transfected cells were normalized to $\mathrm{NC}$, indicating plasmid transfection in cells. Error bars represent standard error. The symbols * and ** indicate $\mathrm{p}<0.05$ and 0.01 , respectively. Scale bar: 100 um.

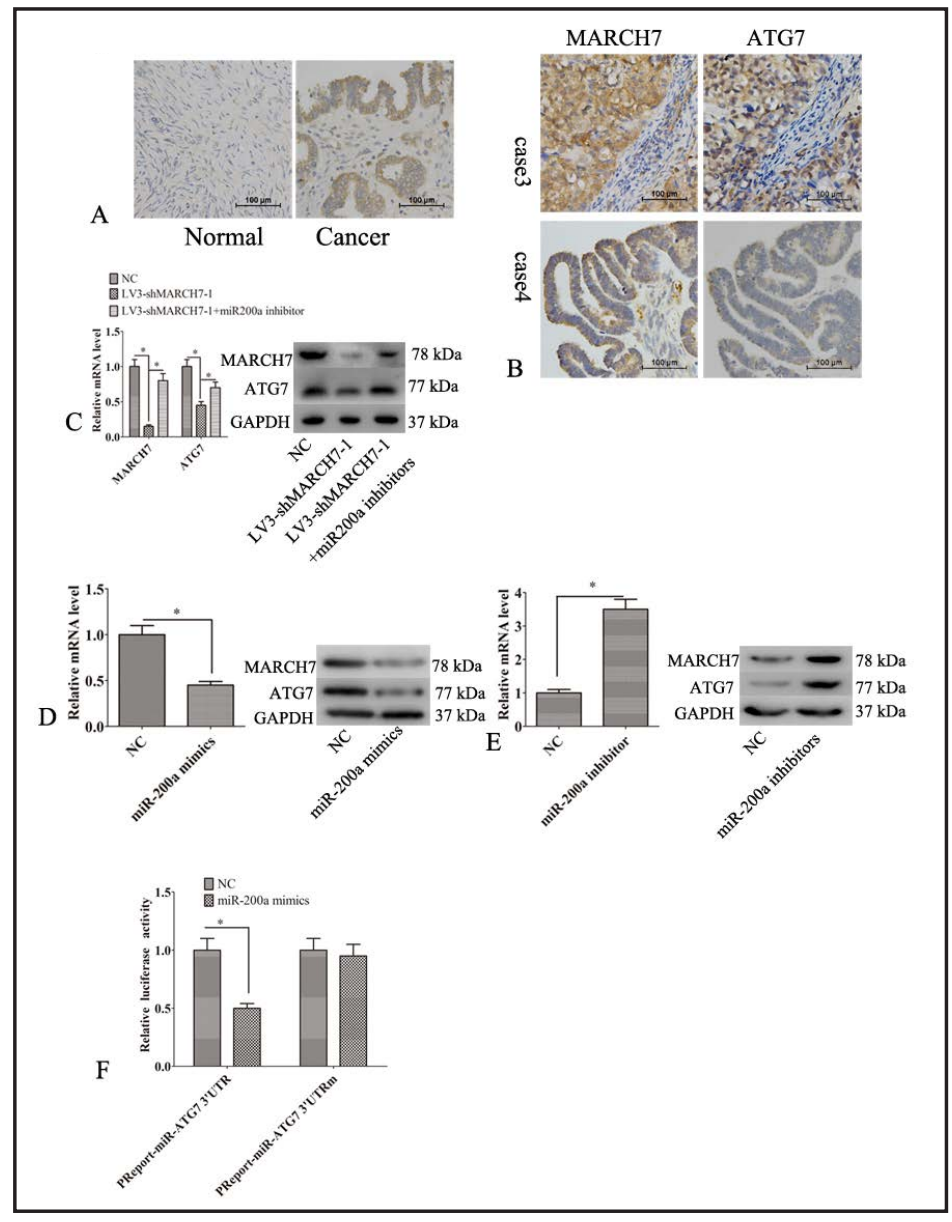




\section{Cellular Physiology Cell Physiol Biochem 2018;47:654-666 \begin{tabular}{l|l|l} 
DOI: 10.1159/000490020 & $\begin{array}{l}\text { O } 2018 \text { The Author(s). Published by S. Karger AG, Basel } \\
\text { wwww.karger.com/cpb }\end{array}$
\end{tabular}}

Hu et al.: MARCH7 Promotes Autophagy and Invasion in Ovarian Cancer

and silencing of ATG7 downregulated MALAT1. However, this regulation was abolished when miR-200a was inhibited (Fig. 3A and B). Our data indicated that MALAT1, MARCH7 and ATG7 reciprocally regulated each other by miR-200a.

\section{MARCH7 interacted and regulated TGFBR2}

Our previous study showed that TGF- $\beta 1$ increased the expression of MARCH7 [9]. So, we hypothesized the involvement of MARCH7 in TGF- $\beta$-smad2/3 pathway. Luciferase reporter assay of TGF- $\beta$-smad $2 / 3$ pathway showed increased activity by ectopic expression of MARCH7 in ovarian cancer A2780 cells infected with LV5-MARCH7 (Fig. 4A). The silencing of MARCH7 in SKOV3 cells caused a marked decrease in TGF- $\beta$-smad2/3 luciferase activity (Fig. 4B). The expression of p-smad2 was also regulated by MARCH7 (Fig. 4C and D).

Fig. 3. MALAT1 reciprocally regulated ATG7 dependent miR-200a. (A) ATG7 was inhibited when silencing MALAT1. This regulation was abolished when transfected with miR-200a inhibitors. (B) MALAT1 was inhibited when silencing ATG7. This regulation was abolished when transfected with miR-200a inhibitors. Error bars represent standard error. The symbols $*$ and $* *$ indicate $\mathrm{p}<0.05$ and

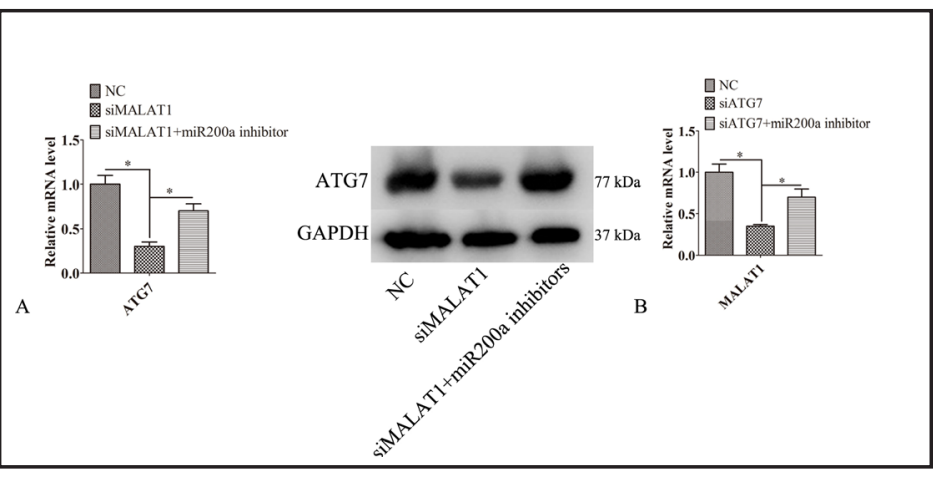
0.01 , respectively. Scale bar: 100 um.

Fig. 4. MARCH7 interacted and regulated TGFBR2. Luciferase reported activity of TGFB1SMAD2/3 signaling pathway was detected in ectopically expressed MARCH7 in A2780 cells infected with LV5-MARCH7. (B). TGFB1SMAD2/3 signaling pathway luciferase reporter activity was detected in LV3-shMARCH7-1, LV3shMARCH7-2 and LV3-NC infected SKOV3 cells. (C) Ovarian cancer SKOV3 cells were transfected with LV3-NC (NC), LV3-shMARCH7-1 or

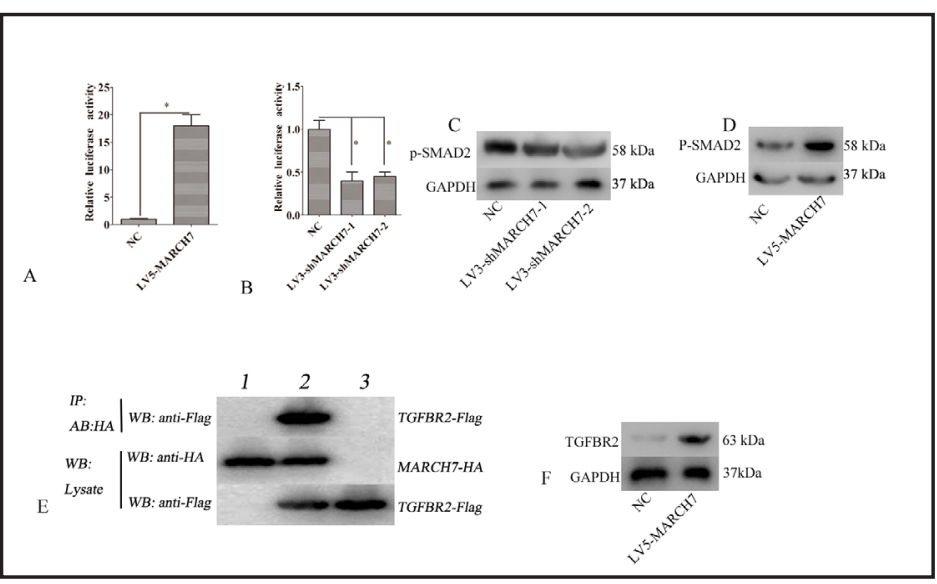
LV3-shMARCH7-2. The protein ex-

pression of P-SAMD2 was detected by western blotting. (D) Ovarian cancer A2780 cells were transfected with LV5-GFP (NC) or LV5-MARCH7. The protein expression of P-SAMD2 was detected by western blotting. (E) Interaction between MARCH7 and TGFBR2 was identified by Co-IP assay. Cells were co-transfected with Flag-TGFBR2 and HA-MARCH7, and control group was established simultaneously, cells were then harvested $24 \mathrm{~h}$ later. Anti-HA antibodies were used to pull the interaction protein. Then, they were detected by anti-Flag antibodies. Results showed that Flag bands could not be detected in the cells transfected with Flag-TGFBR2 (lane 3) or HA-MARCH7 (lane 1) only. However, it can be detected in cells co-transfected with both Flag-TGFBR2 and HA-MARCH7 (lane 2), indicating the existence of interaction between MARCH7 and TGFBR2 in vivo. (F) Ovarian cancer A2780 cells were transfected with LV5-GFP (NC) or LV5-MARCH7. The protein expression of TGFBR2 was detected by western blotting. Error bars represent standard error. The symbols $*$ and ${ }^{* *}$ indicate $\mathrm{p}<0.05$ and 0.01 , respectively.

\section{KARGER}


Fig. 5. TGF- $\beta-S M A D 2 / 3$ pathway regulated MARCH7 and ATG7 though directly regulating MALAT1. (A) The expression of MALAT1, MARCH7 and ATG7 levels in SKOV3 cells was regulated by TGFB1. (B) TGF- $\beta 1$ increased the expression of MALAT1, MARCH7 and ATG7. In contrast, MALAT1 silencing reversed the upregulation of MARCH7 and ATG7 induced by TGF- $\beta 1$. (C) The effect of YY1 on the expression of luciferase reporter driven by the upstream sequence of MALAT1 with or without
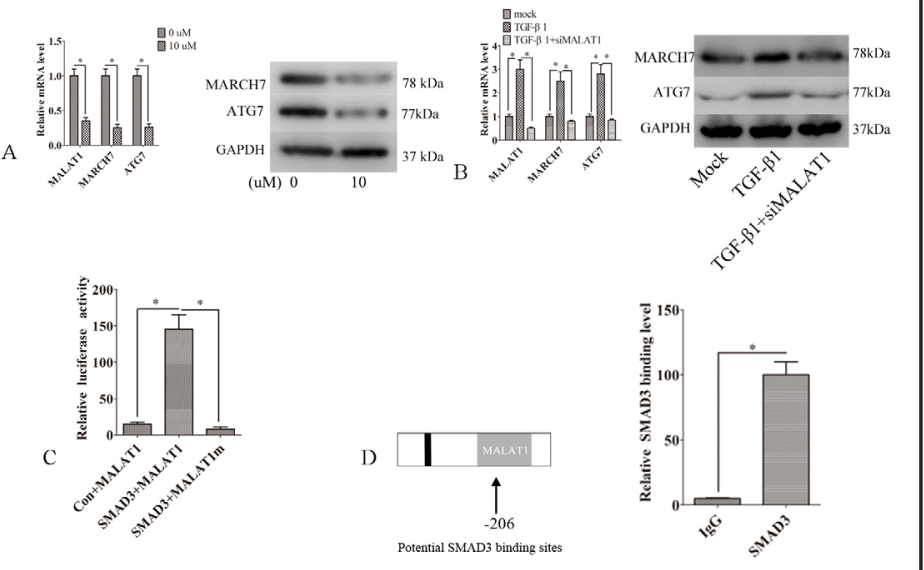

SMAD3-binding sites (M: site was

mutated) were determined by the luciferase activity assay. (D) The putative binding sites between MALAT1 and SMAD3. The relative binding level was detected by chip assay.

Next, we hypothesized that MARCH7 may interact with TGFBR1, TGFBR2, and smad2 or smad3. Co-immunoprecipitation assay confirmed that MARCH7 interacted with TGFBR2 (Fig. 4E). We also observed that the ectopic expression of MARCH7 increased the protein expression of TGFBR2 (Fig. 4F). These results suggest that MARCH7 may regulate TGF- $\beta$ $\operatorname{smad} 2 / 3$ pathway.

TGF- $\beta$-SMAD2/3 pathway regulated MARCH7 and ATG7 by directly regulating MALAT1

Next, we investigated whether TGF- $\beta$-SMAD2/3 pathway regulated MALAT1, MARCH7 and ATG7. Our data showed that LY2109761 inhibited MALAT1, MARCH7 and ATG7 expression levels (Fig. 5A). Activation of TGF- $\beta-S m a d 2 / 3$ pathway using TGF- $\beta 1$ increased the expression of MALAT1, MARCH7 and ATG7. In contrast, MALAT1 silencing reversed the upregulation of MARCH7 and ATG7 induced by TGF- $\beta 1$ (Fig. 5B). Interestingly, there are three potential Smad3 binding sites in the upstream of MALAT1 gene, indicating that smad3 may directly regulate MALAT1 (http://rna.sysu.edu.cn/chipbase/). We found that smad3 increased the activity of luciferase reporter driven by MALAT1 promoter fragment with smad3 binding sites. In contrast, smad3 exhibited little effect on the expression of luciferase reporter regulated by MALAT1 promoter with mutated smad3 binding site (Fig. 5C). Chromatin immunoprecipitation (ChIP) analysis further revealed the binding of smad3 to the binding site. These results confirmed MALAT1 as the direct target of smad3 (Fig. 5D).

\section{MARCH7 regulated TGF- $\beta$ induced autophagy, migration and invasion}

TGF- $\beta$-SMAD2/3 pathway regulated autophagy, migration and invasion. We determined whether MARCH7 regulated TGF- $\beta$ induced autophagy, migration and invasion. Indeed, our data showed that ectopic expression of MARCH7 promoted TGF- $\beta$ induced autophagy, migration and invasion in A2780 cells, whereas MARCH7 silencing inhibited TGF- $\beta$ induced autophagy, migration and invasion in SKOV3 cells (Fig. 6A).

We found that cell migration and invasion ability was decreased in LV3-shMARCH7-1 infected SKOV3 cells compared to LV3-NC infected SKOV3 cells in vivo. The expression of ATG7, TGFBR2 and MALAT1 in tumors from Lv3-shMARCH7-1 infected group was lower than that in the LV3-NC infected group (Fig. 6B).

miR-200a regulated TGF- $\beta$ induced autophagy, migration and invasion by targeting MARCH7

MiR-200a mimic inhibited TGF- $\beta$ induced autophagy, migration and invasion. The phenotypes can partially restore the expression of miR-200a resistant MARCH7. These data indicated that MARCH7 was a direct target of miR-200a (Fig. 7). 


\section{Cellular Physiology and Biochemistry}

Fig. 6. MARCH7 regulated TGF- $\beta$ induced autophagy, migration and invasion. (A) SKOV3 cells transfected with mRFP-GFP-LC3 and MARCH7 or control siRNAs. After $24 \mathrm{~h}$, TGFB1 was added at a concentration of $10 \mathrm{ng} / \mathrm{ml}$. mRFP-GFPLC3 distribution in SKOV3 cells was analyzed by confocal microscopy after TGFB1 treatment for $24 \mathrm{~h}$. The LC3 dots were quantified using the image pro-plus 6.0 software. All experiments were repeated 3 times and the representative results are shown. A2780 cells transfected with mRFP-GFP-LC3 and LV5-MARCH7 or LV5-GFP. After $24 \mathrm{~h}$, TGFB1 was added at a concentration of $10 \mathrm{ng} / \mathrm{ml}$. mRFPGFP-LC3 distribution was analyzed by confocal microscopy after TGFB1 treatment for $24 \mathrm{~h}$. The LC3 dots were quantified using the image pro-plus 6.0 software. All experiments were repeated 3 times

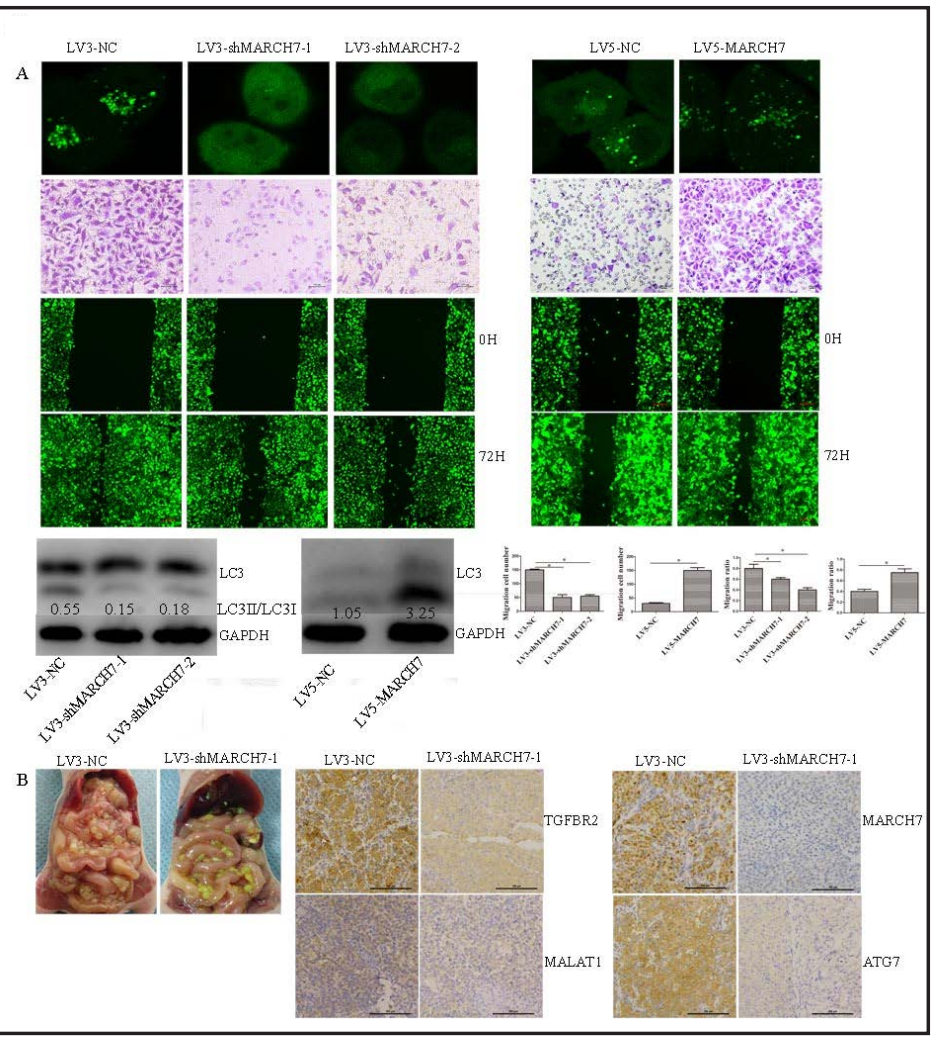
and the representative results are shown. The migration ability of ovarian cancer SKOV3 and A2780 cells was detected by wound healing assay. SKOV3 cells were infected with LV3-shMARCH7-1, LV3-shMARCH7-2 and LV3-NC, and the A2780 cells were infected with LV5-GFP and LV5-MARCH7. After $48 \mathrm{~h}$, the puromycin was added at a concentration of $2.5 \mathrm{ug} / \mathrm{ml}$. After $72 \mathrm{~h}$, the migration assays were carried out in the presence of TGFB1 (10 ng/ml). The invasive ability of ovarian cancer SKOV3 and A2780 cells was detected by Matrigel invasion assay. The SKOV3 cells were infected with LV3-shMARCH7-1, LV3-shMARCH7-2 and LV3-NC, and the A2780 cells were infected with LV5-GFP and LV5-MARCH7. After $48 \mathrm{~h}$, the puromycin was added at a concentration of 2.5ug/ $\mathrm{ml}$. After $72 \mathrm{~h}$, the invasion assays were carried out in the presence of TGFB1 (10 ng/ml). (B). Silencing of MARCH7 inhibited pelvic peritoneal metastasis in nude mice model. Immunohistochemical analysis of MARCH7, ATG7, and TGFBR2 expressions were performed on tumor xenografts. The expression of MALAT1 was detected by ISH. Representative images are shown (original magnification $\times 200$ ). Error bars represent standard error. The symbols * and ${ }^{* *}$ indicate $\mathrm{p}<0.05$ and 0.01 , respectively. Scale bar: 100 um.

MARCH7 promoted TGF- $\beta$ induced autophagy, migration and invasion through MALAT1, ATG7 and TGF- $\beta$-SMAD2/3 pathway

MARCH7 interacted with MALAT1, ATG7 and TGFBR2. So, we investigated whether MARCH7 promoted TGF- $\beta$ induced autophagy, migration and invasion required for MALAT1, ATG7 and TGF- $\beta$-SMAD2/3 pathway. Results revealed that cellular migration, invasion and autophagy were decreased in LV5-MARCH7 infected A2780 cells with inhibition of TGF- $\beta$ SMAD2/3 pathway or knockdown of MALAT1 or ATG7 (Fig. 8A). These results indicated that MARCH7 regulated TGF- $\beta$ induced autophagy, migration and invasion required for MALAT1, ATG7 and TGF- $\beta$-SMAD2/3 pathway (Fig. 8B).

\section{Discussion}

In the present study, we found E3 ubiquitin ligase MARCH7 interaction with MALAT1, regulating ATG7 through competing with miR-200a. MARCH7 promotes TGF- $\beta$ mediated autophagy, invasion and metastasis in ovarian carcinoma. We also found that TGF- $\beta$ - 


\section{Cellular Physiology Cell Physiol Biochem 2018;47:654-666 \begin{tabular}{l|l} 
DOI: 10.1159/000490020 & O 2018 The Author(s). Published by S. Karger AG, Basel \\
www.karger.com/cpb
\end{tabular}}

SMAD2/3 regulated MARCH7 and ATG7 by directly modulating MALAT1.

Autophagy has dual roles in cancer, as a tumor suppressor by degrading the damaged proteins and organelles and as a mechanism of cell survival, enabling the promotion of growth tumors $[15,16]$. ATG7 dependent autophagy protects breast cancer cell lines against mitoquinone-induced oxidative stress [17]. Knockdown of ATG7 strongly enhanced the apoptosis induced by various anticancer agents including tyrosine kinase inhibitors [18-20]. Recent studies showed that autophagy may promote carcinoma cell invasion and correlate with poor prognosis in cholangiocarcinoma $[21,22]$. In this study, we found that blockage of autophagy by downregulation of ATG7 or MARCH7 expression led to the inhibition of TGF- $\beta$ induced cellular migration and invasion. Our results were inconsistent with the previous report, indicating that inhibition of autophagy impairs tumor cell invasion [23]. MiR-200c inhibits autophagy and enhances radiosensitivity in breast cancer cells [24]. MiR-200 family inhibits the migration and invasion ability of ovarian cancer cells by inducing mesenchymal to epithelial

Fig. 8. MARCH7 promoted TGF- $\beta$ induced autophagy, migration and invasion required for MALAT1, ATG7 and TGF- $\beta$-SMAD2/3 pathway. (A) The A2780 cells were infected with LV5MARCH7. After $48 \mathrm{~h}$, the puromycin was added at a concentration of $2.5 \mathrm{ug} / \mathrm{ml}$. The cells were transfected with ATG7 siRNA, MALAT1 siRNA or LY2109761 was added (10uM) for $48 \mathrm{~h}$. Then, the autophagy, migration and invasion were determined. (B) The graphic abstract for the significance of MARCH7 pathway (miR-200a, SMAD3, MARCH7, ATG7, and MALAT1). Error bars represent standard error. The symbols $*$ and ${ }^{* *}$ indicate $\mathrm{p}<0.05$ and 0.01 , respectively. Scale bar: 100 um.

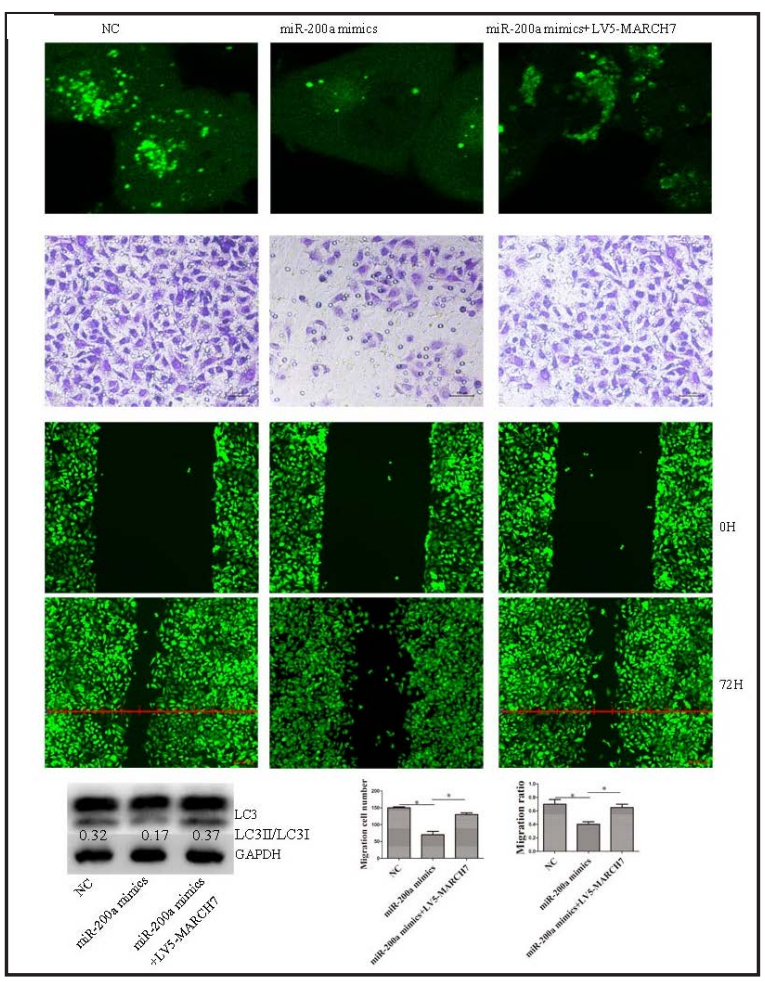

Fig. 7. miR-200a regulated TGF- $\beta$ induced autophagy, migration and invasion by targeting MARCH7. SKOV3 cells were transfected with miR-200a or miR-200a+LV5MARCH7. Then, the autophagy, migration and invasion were determined. Error bars represent standard error. The symbols * and ** indicate $\mathrm{p}<0.05$ and 0.01 respectively. Scale bar: 100 um.

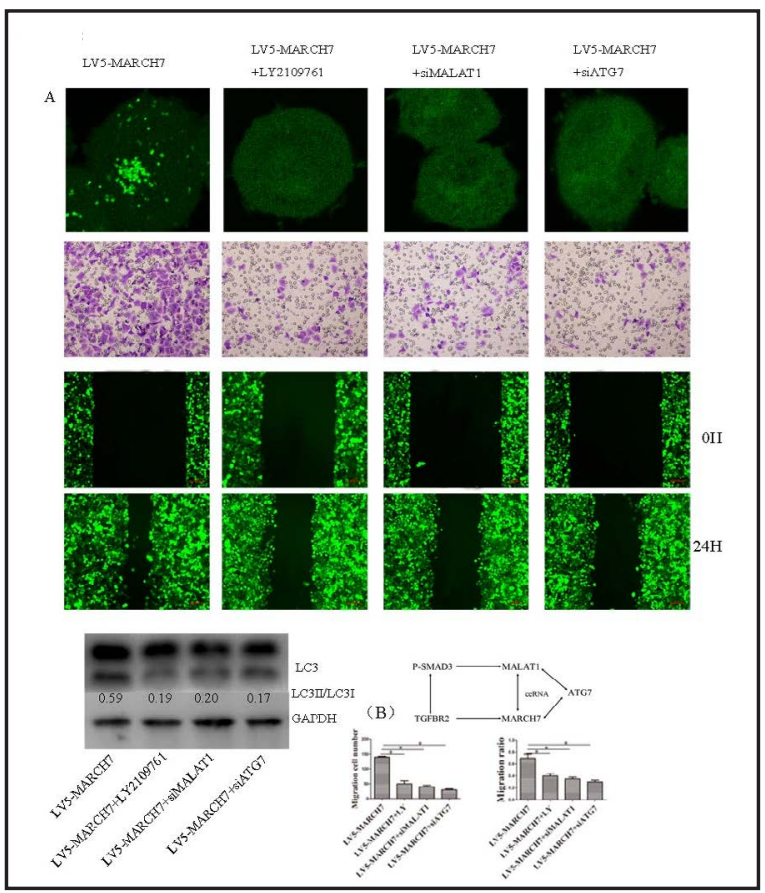


transition (MET) [25]. In our study, miR-200a inhibited autophagy, migration and invasion in ovarian cancer. However, the phenotypes can partially restore the expression of miR-200a resistant MARCH7. This data confirmed the role of MARCH7 in the regulation of cellular autophagy, migration and invasion.

Metastasis-associated lung adenocarcinoma transcript 1 (MALAT1), a long non-coding RNA (lncRNA), was up-regulated in many tumors and associated with cancer cell metastasis and recurrence $[26,27]$. Recent study demonstrated that TGF- $\beta$-induced upregulation of MALAT1 promoted bladder cancer metastasis [28]. Our study demonstrated TGF- $\beta$ induced upregulation of MALAT1 and knockdown of MALAT1 inhibited TGF- $\beta$-induced autophagy, migration and invasion. Silencing of MALAT1 by miR-101 and miR-217 inhibits proliferation, migration, and invasion of esophageal squamous cell carcinoma cells [29]. In this study, we found that miR-101 and miR-200a inhibited the expression of MALAT1. Previous studies confirmed that miR-101 and miR-200a inhibited autophagy, migration and invasion [24, 30, 31]. MALAT1 regulated autophagy, migration and invasion through miR-101 and miR-200a. Our previous study has demonstrated MARCH7 as a direct target of miR-101[9]. Our study found that MARCH7 and MALAT1 regulated each other, indicating that MARCH7 regulated MALAT1 partly by depending on miR-101. LncRNA could regulate the target protein mainly by directly binding to the target protein and posttranscriptional modification through sponge miRNAs [32, 33]. MALAT1 interacted with MARCH7 by miR-200a, and MARCH7 regulated ATG7 by competing for miR-200a. MALAT1 regulated MARCH7 and ATG7 through sponging miR-200a.

TGF- $\beta$ activates SMAD2/3 through a heteromeric receptor complex that consists of type I (TGFBR1) and type II (TGFBR2) components [34, 35]. Knockdown of TGFBR2 was reported to inhibit the invasion of glioma cells [36]. We showed that MARCH7 directly interacted with and regulated TGFBR2. TGF- $\beta$-SMAD2/3 pathway inhibitor inhibited MARCH7 and elevated cellular autophagy, migration and invasion. This data demonstrated that MARCH7 promoted cellular autophagy, migration and invasion through TGF- $\beta$-SMAD2/3 pathway. Knockdown of TGFBR2 or inhibition of TGF- $\beta$-SMAD2/3 pathway remarkably decreased the expression of MARCH7, MALAT1 and ATG7. SMAD2/3 directly binds to and regulates MALAT1. These results indicated that TGF- $\beta$-SMAD2/3 pathway regulated MARCH7 and ATG7 through MALAT1.

In conclusion, this study confirmed that MARCH7 is a tumor promoting gene in human ovarian cancer, and involved in TGF- $\beta$-smad $2 / 3$ pathway. This also suggested that MARCH7 may be a potential therapeutic target in epithelial ovarian cancer.

\section{Acknowledgements}

We thank members of our laboratory for technical help and stimulating discussions. This work was supported by the Natural Science Foundation of Chongqing (CSTC 2012JJB10030); the National Science Foundation of China (81702557) ; and the National Science Foundation of China (81501220).

\section{Disclosure Statement}

The authors declare that there is no potential conflict of interests.

\section{References}

$\rightarrow 1$ Bierie B, Moses HL: Tumour microenvironment: TGFbeta: the molecular Jekyll and Hyde of cancer. Nat Rev Cancer 2006;6:506-520.

2 Leivonen SK, Kahari VM: Transforming growth factor-beta signaling in cancer invasion and metastasis. Int J Cancer 2007;121:2119-2124. 


\section{Cellular Physiology Cell Physiol Biochem 2018;47:654-666 \begin{tabular}{l|l} 
DOI: 10.1159/000490020 & $\begin{array}{l}\text { O } 2018 \text { The Author(s). Published by S. Karger AG, Basel } \\
\text { www.karger.com/cpb }\end{array}$
\end{tabular}}

Hu et al.: MARCH7 Promotes Autophagy and Invasion in Ovarian Cancer

- 3 Geng J, Fan J, Ouyang Q Zhang X, Zhang X, Yu J, Xu Z, Li Q, Yao X, Liu X, Zheng J: Loss of PPM1A expression enhances invasion and the epithelial-to-mesenchymal transition in bladder cancer by activating the TGFbeta/Smad signaling pathway. Oncotarget 2014;5:5700-5711.

4 Hsu HY, Lin TY, Wu YC, Tsao SM, Hwang PA, Shih YW, Hsu J: Fucoidan inhibition of lung cancer in vivo and in vitro : role of the Smurf2-dependent ubiquitin proteasome pathway in TGFbeta receptor degradation. Oncotarget 2014;5:7870-7885.

-5 Simbolo M, Fassan M, Ruzzenente A, Mafficini A, Wood LD, Corbo V, Melisi D, Malleo G, Vicentini C, Malpeli G, Antonello D, Sperandio N, Capelli P, Tomezzoli A, Iacono C, Lawlor RT, Bassi C, Hruban RH, Guglielmi A, Tortora G, de Braud F, Scarpa A: Multigene mutational profiling of cholangiocarcinomas identifies actionable molecular subgroups. Oncotarget 2014;5:2839-2852.

6 Ungefroren H, Sebens S, Giehl K, Helm O, Groth S, Fandrich F, Rocken C, Sipos B, Lehnert H, Gieseler F: Rac1b negatively regulates TGF-beta1-induced cell motility in pancreatic ductal epithelial cells by suppressing Smad signalling. Oncotarget 2014;5:277-290.

7 Gajewska M, Gajkowska B, Motyl T: Apoptosis and autophagy induced by TGF-B1 in bovine mammary epithelial BME-UV1 cells. J Physiol Pharmacol 2005;56:S143-157.

8 Kiyono K, Suzuki HI, Matsuyama H, Morishita Y, Komuro A, Kano MR, Sugimoto K, Miyazono K: Autophagy is activated by TGF-beta and potentiates TGF-beta-mediated growth inhibition in human hepatocellular carcinoma cells. Cancer Res 2009;69:8844-8852.

-9 Hu J, Meng Y, Yu T, Hu L, Mao M: Ubiquitin E3 Ligase MARCH7 promotes ovarian tumor growth. Oncotarget 2015.

10 Au CW, Siu MK, Liao X, Wong ES, Ngan HY, Tam KF, Chan DC, Chan QK, Cheung AN: Tyrosine kinase B receptor and BDNF expression in ovarian cancers - Effect on cell migration, angiogenesis and clinical outcome. Cancer Lett 2009;281:151-161.

11 Wu H, Li Z, Yang P, Zhang L, Fan Y, Li Z: PKM2 depletion induces the compensation of glutaminolysis through beta-catenin/c-Myc pathway in tumor cells. Cell Signal 2014;26:2397-2405.

12 Wang M, Zhang Y, Wang T, Zhang J, Zhou Z, Sun Y, Wang S, Shi Y, Luan X, Zhang Y, Wang Y, Wang Y, Zou Z, Kang L, Liu H: The USP7 Inhibitor P5091 Induces Cell Death in Ovarian Cancers with Different P53 Status. Cell Physiol Biochem 2017;43:1755-1766.

13 Zhang Y, Zhao FJ, Chen LL, Wang LQ Nephew KP, Wu YL, Zhang S: MiR-373 targeting of the Rab22a oncogene suppresses tumor invasion and metastasis in ovarian cancer. Oncotarget 2014;5:12291-12303.

14 Zeng R, Zhang R, Song X, Ni L, Lai Z, Liu C, Ye W: The long non-coding RNA MALAT1 activates Nrf2 signaling to protect human umbilical vein endothelial cells from hydrogen peroxide. Biochem Biophys Res Commun 2018;495:2532-2538.

15 Chen P, Cescon M, Bonaldo P: Autophagy-mediated regulation of macrophages and its applications for cancer. Autophagy 2014;10:192-200.

16 Zhang SF, Wang XY, Fu ZQ, Peng QH, Zhang JY, Ye F, Fu YF, Zhou CY, Lu WG, Cheng XD, Xie X: TXNDC17 promotes paclitaxel resistance via inducing autophagy in ovarian cancer. Autophagy 2015:0.

17 Gonzalez Y, Aryal B, Chehab L, Rao VA: Atg7- and Keap1-dependent autophagy protects breast cancer cell lines against mitoquinone-induced oxidative stress. Oncotarget 2014;5:1526-1537.

-18 Carew JS, Medina EC, Esquivel JA 2nd, Mahalingam D, Swords R, Kelly K, Zhang H, Huang P, Mita AC, Mita MM, Giles FJ, Nawrocki ST: Autophagy inhibition enhances vorinostat-induced apoptosis via ubiquitinated protein accumulation. J Cell Mol Med 2010;14:2448-2459.

19 Han W, Sun J, Feng L, Wang K, Li D, Pan Q Chen Y, Jin W, Wang X, Pan H, Jin H: Autophagy inhibition enhances daunorubicin-induced apoptosis in K562 cells. PLoS One 2011;6:e28491.

20 Wang Y, Ding L, Wang X, Zhang J, Han W, Feng L, Sun J, Jin H, Wang XJ: Pterostilbene simultaneously induces apoptosis, cell cycle arrest and cyto-protective autophagy in breast cancer cells. Am J Transl Res 2012;4:4451.

21 Nitta T, Sato Y, Ren XS, Harada K, Sasaki M, Hirano S, Nakanuma Y: Autophagy may promote carcinoma cell invasion and correlate with poor prognosis in cholangiocarcinoma. Int J Clin Exp Pathol 2014;7:4913-4921.

22 Zhan Z, Xie X, Cao H, Zhou X, Zhang XD, Fan H, Liu Z: Autophagy facilitates TLR4- and TLR3-triggered migration and invasion of lung cancer cells through the promotion of TRAF6 ubiquitination. Autophagy 2014;10:257-268.

23 Macintosh RL, Timpson P, Thorburn J, Anderson KI, Thorburn A, Ryan KM: Inhibition of autophagy impairs tumor cell invasion in an organotypic model. Cell Cycle 2012;11:2022-2029. 


\section{Cellular Physiology Cell Physiol Biochem 2018;47:654-666 \begin{tabular}{l|l|l} 
DOI: 10.1159/000490020 & O 2018 The Author(s). Published by S. Karger AG, Basel \\
www.karger.com/cpb
\end{tabular}}

Hu et al.: MARCH7 Promotes Autophagy and Invasion in Ovarian Cancer

-24 Sun Q, Liu T, Yuan Y, Guo Z, Xie G, Du S, Lin X, Xu Z, Liu M, Wang W, Yuan Q Chen L: MiR-200c inhibits autophagy and enhances radiosensitivity in breast cancer cells by targeting UBQLN1. Int J Cancer 2015;136:1003-1012.

25 Chen J, Wang L, Matyunina LV, Hill CG, McDonald JF: Overexpression of miR-429 induces mesenchymal-toepithelial transition (MET) in metastatic ovarian cancer cells. Gynecol Oncol 2011;121:200-205.

-26 Tee AE, Ling D, Nelson C, Atmadibrata B, Dinger ME, Xu N, Mizukami T, Liu PY, Liu B, Cheung B, Pasquier E, Haber M, Norris MD, Suzuki T, Marshall GM, Liu T: The histone demethylase JMJD1A induces cell migration and invasion by up-regulating the expression of the long noncoding RNA MALAT1 Oncotarget 2014;5:1793-1804.

-27 Hirata H, Hinoda Y, Shahryari V, Deng G, Nakajima K, Tabatabai ZL, Ishii N, Dahiya R: Long noncoding RNA MALAT1 promotes aggressive renal cell carcinoma through Ezh2 and interacts with miR-205 Cancer Res 2015.

28 Fan Y, Shen B, Tan M, Mu X, Qin Y, Zhang F, Liu Y: TGF-beta-induced upregulation of malat1 promotes bladder cancer metastasis by associating with suz12 Clin Cancer Res 2014;20:1531-1541.

29 Wang X, Li M, Wang Z, Han S, Tang X, Ge Y, Zhou L, Zhou C, Yuan Q, Yang M: Silencing of Long Noncoding RNA MALAT1 by miR-101 and miR-217 Inhibits Proliferation, Migration, and Invasion of Esophageal Squamous Cell Carcinoma Cells. J Biol Chem 2015;290:3925-3935.

-30 Frankel LB, Wen J, Lees M, Hoyer-Hansen M, Farkas T, Krogh A, Jaattela M, Lund AH: microRNA-101 is a potent inhibitor of autophagy. EMBO J 2011;30:4628-4641.

-31 Luo C, Merz PR, Chen Y, Dickes E, Pscherer A, Schadendorf D, Eichmuller SB: MiR-101 inhibits melanoma cell invasion and proliferation by targeting MITF and EZH2. Cancer Lett 2013;341:240-247.

-32 Mathieu EL, Belhocine M, Dao LT, Puthier D, Spicuglia S: [Functions of lncRNA in development and diseases]. Med Sci (Paris) 2014;30:790-796.

-33 Yang G, Lu X, Yuan L: LncRNA: a link between RNA and cancer. Biochim Biophys Acta 2014;1839:10971109.

34 Grady WM: Transforming growth factor-beta, Smads, and cancer. Clin Cancer Res 2005;11:3151-3154.

-35 Bharathy S, Xie W, Yingling JM, Reiss M: Cancer-associated transforming growth factor beta type II receptor gene mutant causes activation of bone morphogenic protein-Smads and invasive phenotype. Cancer Res 2008;68:1656-1666.

36 Wesolowska A, Kwiatkowska A, Slomnicki L, Dembinski M, Master A, Sliwa M, Franciszkiewicz K, Chouaib S, Kaminska B: Microglia-derived TGF-beta as an important regulator of glioblastoma invasion--an inhibition of TGF-beta-dependent effects by shRNA against human TGF-beta type II receptor. Oncogene 2008;27:918930. 